\title{
Preoperative Tranexamic Acid in conjunction with Crystalloid Co-load improves the outcome of Elective Cesarean Section under spinal anesthesia
}

\author{
Original \\ Article \\ Ibrahiem Swedan', Tamer Mahmoud Assar ${ }^{1}$, Elsayed Abdelazim², and Mohamed \\ Fouad ${ }^{2}$
}

${ }^{1}$ Department of Obstetrics \& Gynecology and ${ }^{2}$ Department of Anesthesia, Faculty of Medicine, Banha University, Egypt

\begin{abstract}
Objective: To evaluate therapeutic yield of tranexamic acid (TXA) injection and fluid co-load on the outcome of cesarean section (CS) conducted under spinal anesthesia

Study Design : Prospective randomized comparative study.

Patients and Methods: The current included 174 primipara scheduled for elective CS under spinal anesthesia. Intravenous (IV) fluid co-load ( $15 \mathrm{ml} / \mathrm{kg}$ warm lactated ringer solution) started as fast drip during and continued after spinal anesthesia. Patients were randomly allocated into two equal groups: Group TXA received a loading IV dose of 500 mg TXA 20 minutes before surgery followed by continuous TXA infusion at rate of $1 \mathrm{mg} / \mathrm{kg} / \mathrm{min}$ till end of surgery. Group C did not receive prophylactic TXA, but both groups received a booster dose of 2 gm TXA if required. Patients were monitored for the frequency and severity of hypotension and dose of ephedrine used. Amount of bleeding since skin incision till 2-hours postpartum (PP), the frequency of patients had stopped PP bleeding till 2-hr PP and the total dose of oxytocin drugs and booster doses of TXA were recorded.

Results: Hypotension was recorded in 53 patients (30.5\%); only 12 patients had blood pressure $<80 \mathrm{mmHg}$. The lowest blood pressure, frequency of hypotension, time of occurrence and duration of hypotension and the used dose of ephedrine showed non-significant difference between both studied groups. The amount of perioperative bleeding was significantly lower in TXA group compared to control group. Twenty parturient had continued PP bleeding for more than 2 hours PP with significantly $(\mathrm{p}=0.038)$ lower frequency of bleeders in TXA group. All women had continued bleeding received booster doses of TXA with a significantly higher dose in control group compared to TXA group.

Conclusion: Preoperative administration of TXA significantly reduced perioperative bleeding with significant reduction of consumption of TXA booster doses and utrotonics without affecting safety. Fluid coloading allowed reduction of the frequency and extent of hypotension concomitant with spinal anesthesia.
\end{abstract}

Received: 5th May 2017, Accepted: 14 July 2017

Key Words: Cesarean section, , Fluid co-load, Tranexamic acid, Spinal anesthesia

Corresponding Author: Tamer Mahmoud Assar, Faculty of Medicine, Banha University, Banha, Alkalubia, Egypt, Tel.: +20 1273580301, E-mail: tamer.assar2000@gmail.com

ISSN: 2090-7625, November 2017, Vol.7, No. 4

\section{INTRODUCTION}

Uterine blood flow is directly dependent on maternal blood pressure and maternal hypotension may have deleterious effects on the fetus. If sustained, it may lead to fetal endangerment and possibility of death. Therefore, maternal hypotension should always be treated. Despite the shorter duration of anesthesia associated with caesarean delivery, the potential for vasodilation, core-to-peripheral redistribution of body heat, and a resulting state of mild maternal hypothermia exists. Furthermore, it is well known that maternal body temperature is associated with neonatal body temperature. It is well known that ephedrine has thermogenic properties related to its beta stimulating properties. Also the vasoconstriction effect of ephedrine may have prevented the internal core-to-peripheral redistribution of heat ${ }^{[1-4]}$.

Postpartum hemorrhage causes hospital morbidity; approximately $1 \%$ of women with spontaneous vaginal deliveries require transfusion, but the figure increases to $5 \%$ or $6 \%$ for women with instrumental deliveries or 
caesarean sections. The risk of infection from transfused blood is considerably higher especially in countries that do not screen all blood for transfusion, and other adverse reactions related to blood transfusion are common, irrespective of country development ${ }^{[5-7]}$.

Multiple studies reported the efficacy of tranexamic acid (TXA) used during various surgical procedures requiring blood transfusion or conservation; Sankar et al. ${ }^{[8]}$ compared TXA $(10 \mathrm{mg} / \mathrm{kg})$ as a preoperative bolus followed by $1 \mathrm{mg} / \mathrm{kg}$ as a maintenance intraoperative dose versus placebo during orthognathic surgery and reported statistically significant reduction in blood loss and improved quality of surgical field with TXA. Wind et al. ${ }^{[9]}$ found a statistically significant decrease in blood transfusion rate and in both immediate postoperative and day of discharge hematocrit levels in patients having TXA infusion compared to placebo. Roberts et al. ${ }^{[10]}$ found early administration of TXA safely reduced the risk of death in bleeding trauma patients and is highly cost-effective. Vijay et al. ${ }^{[11]}$ assessed the outcome of TXA given in a bolus dose of $500 \mathrm{mg} 15 \mathrm{~min}$ before surgical incision followed by continuous infusion during major hip and femoral surgeries and concluded that TXA significantly reduces postoperative blood loss and transfusion requirements.

Hypotension is the commonest problem with spinal anesthesia. Perioperative fluid therapy is an option to combat spinal anesthesia induced hypotension; however, there is still debit about type of fluid used either crystalloid or colloid and the timing of administration whether prior to spinal anesthesia as preload or during giving anesthesia as co-load. Multiple studied rejected the preload option and found co-loading is the ideal, another studies found colloid preload and crystalloid coloading did not significantly differ ${ }^{[12,13]}$.

Thus, the current comparative prospective study aimed to evaluate the therapeutic yield of preoperative TXA injection and continuous infusion with fluid co-load on the outcome of cesarean section conducted under spinal anesthesia.

\section{PATIENTS AND METHODS}

The current prospective comparative study was conducted at Departments of Anesthesia and Gynecology \& Obstetrics, Benha University Hospital since Nov 2013 till June 2015. After approval of the study protocol by the Local Ethical Committee and obtaining fully informed written patients' consents, 174 primigravida scheduled for elective CS with uncomplicated pregnancy were included in the study and divided into two equal groups. Demographic data including age, height and weight were determined preoperatively. All study participants underwent laboratory investigations including determination of complete blood count, hemoglobin concentration, serum liver enzymes, prothrombin time and concentration, clotting and bleeding times and serum urea and creatinine. All laboratory investigations were conducted at hospital lab. Exclusion criteria included preeclampsia, any major systemic disease, contraindication to regional anesthesia or allergy to used medications. Also, anemic patients or those had hematological abnormalities or coagulation diathesis, chronic liver or kidney disease were excluded from the study

All parturient received spinal anesthesia using $10 \mathrm{mg}$ bupivacaine $0.5 \%$ injected through spinal needle, $25 \mathrm{G}$, inserted in the L34- or L 45- interspace. Intravenous fluid co-load was given in a dose of $15 \mathrm{ml} / \mathrm{kg}$ warm lactated ringer solution started as fast drip during and continued after spinal anesthesia.

Patients were randomly, using sealed envelops, allocated into two groups: Group TXA received a loading intravenous dose of $500 \mathrm{mg}$ TXA 20 minutes before surgery followed by continuous TXA infusion at rate of $1 \mathrm{mg} / \mathrm{kg} /$ min till the end of surgery. Group $\mathrm{C}$ included patients who did not receive prophylactic TXA. Both groups received a booster dose of $2 \mathrm{gm}$ TXA was given if required during surgery and till 2-hours after the end of surgery.

\section{Patients monitoring included the following:}

1. Blood pressure measures including systolic arterial blood pressure (SAP), diastolic arterial blood pressure (DAP) and mean arterial blood pressure (MAP) were continuously monitored non-invasively till the end of surgery. Hypotension defined as systolic blood pressure $<100 \mathrm{mmHg}$ was treated with ephedrine.

2. Spinal block effectiveness data was monitored for the time of onset of sensory and motor block and the height of maximum sensory block was recorded after $30 \mathrm{~min}$ and the grade of motor block according to modified Bromage scale ${ }^{[14]}$.

3. The effectiveness of the study protocol was evaluated as the following items:

a. Amount of bleeding since skin incision till start of placental separation and since separation of placenta till 2-hours PP as judged by the following method:

- Calculation of the actual blood loss (ABL) was calculated from a modification of the Gross formula (16): $\mathrm{ABL}=\mathrm{BV}[$ Hct (i) - Hct (f)]/ Hct (m) where BV was the blood volume calculated according to the equation: $\mathrm{BV}=$ Body weight $(\mathrm{kg}) \times 70 \mathrm{ml} / \mathrm{kg}(17)$, Hct (i), Hct (f), Hct (m) were the initial, final and mean of initial and final hematocrite values, respectively ${ }^{[18]}$. 
The frequency of patients who had stopped PP bleeding till 2-hr PP.

Rescue uterotonic was provided in form of slow injection of oxytocin $5 \mathrm{IU}$ diluted to $3 \mathrm{ml}$ with normal saline. The frequency of rescue uterotonic used since separation of placenta till 2-hours PP was reported.

The total dose of administration of oxytocin and TXA used as booster dose; if any was determined.

Neonatal outcome evaluation including Apgar score at 1 and 5 minutes, the need for resuscitation and/or admission to neonatal intensive care unit (NICU).

Spinal anesthesia-related side effects; nausea, vomiting, respiratory depression or shivering were recorded and managed. Also, the frequency of side effects related to the use of TXA was recorded.

\section{Statistical analysis}

Sample Power was calculated according to Kraemer \& Thiemann (19) using their proposed figure showed that the sample size for $60 \%$ power would require an $\mathrm{N}$ of 31 /group and $80 \%$ power would require an $\mathrm{N}$ of 51 /group. This hypothesis was documented by Murphy \& Myors (20). Considering excessive bleeding is uncommon event with cesarean section so, from a standard nomogram, a sample size of 87 patient per group was determined to be sufficient to give the trial $80 \%$ power to detect a difference at the 5\% significance level (21). Obtained data were presented as mean $\pm \mathrm{SD}$, ranges, numbers and ratios. Results were analyzed using paired t-test for within group variability and Wilcoxon; ranked test for unrelated data (Z-test) and Chisquare test (X2 test) paired t-test for variability between groups. Statistical analysis was conducted using the SPSS (Version 15, 2006) for Windows statistical package. P value $<0.05$ was considered statistically significant.

\section{RESULTS}

There were no significant statistical differences between both groups as regard maternal age, weight, parity, gestational age, and indications of cesarean sections. All patients passed smooth intraoperative course without complications within a mean duration of surgery 466.6 \pm ; range: 3055 - minutes with no significant difference between both studied groups.

No significant differences were noted between both groups regards the time till onset of sensory block and mean level of maximal sensory block, time till onset and duration of motor block and the distribution of patients among Bromage motor block grades showed nonsignificant difference between studied groups.

Hypotension was recorded in 53 patients $(30.5 \%)$; only 12 patients had severe hypotension with blood pressure $<80 \mathrm{mmHg}$. The lowest blood pressure estimated and the frequency of hypotension showed non-significant $(p>0.05)$ difference between both study groups. Time of occurrence and duration of hypotension and the used dose of ephedrine showed non-significant $(p>0.05)$ difference between both studied groups.

The amount of perioperative bleeding was significantly lower in TXA group compared to control group, irrespective of the method of estimation. Moreover, the mean decrease of hematocrite value was significantly lower in TXA group compared to control group. However, amount of blood loss determined using the gravimetric method was non-significantly higher compared to calculated ABL in both groups, ( Fig. 1)

Twenty parturient had continued PP bleeding for more than 2 hours PP; 14 in control group and 6 in TXA

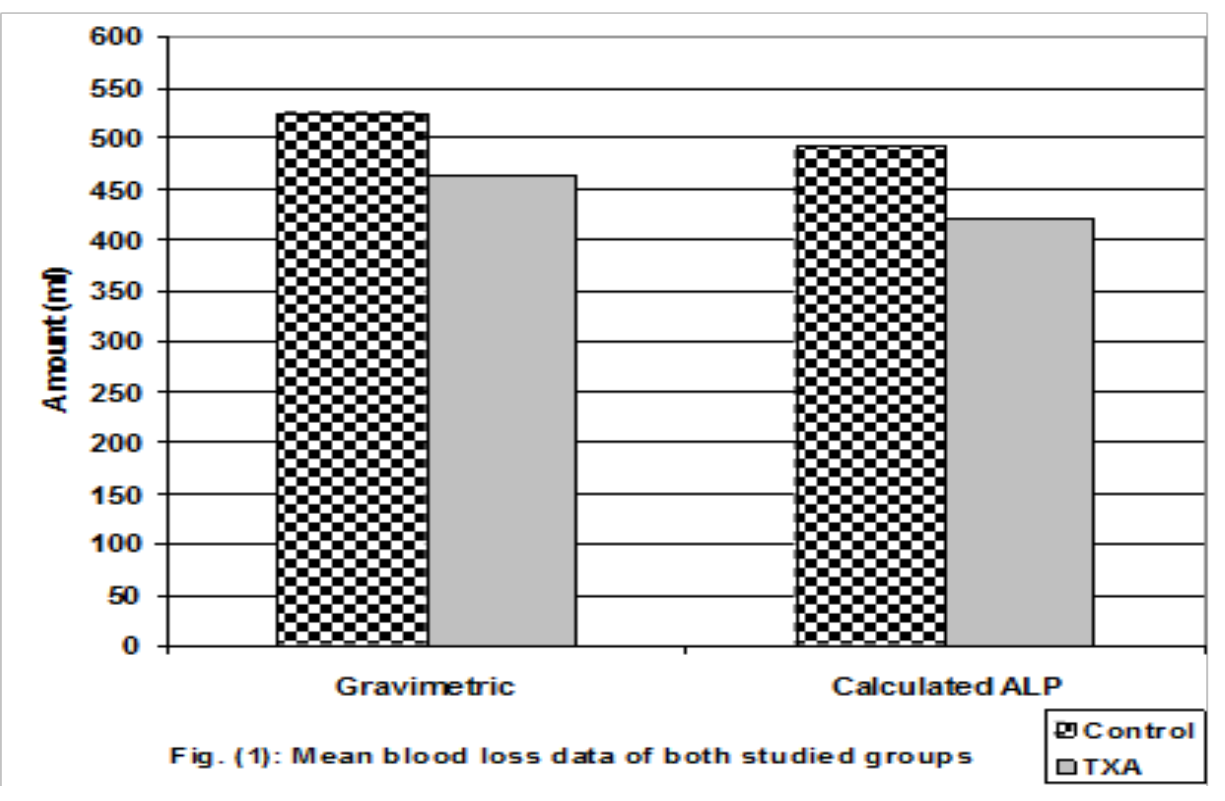


group with significantly $(\mathrm{X} 2=3.285, \mathrm{p}=0.038)$ lower frequency of bleeders in TXA group.

Mean 1-min and 5-min Apgar score was non-
All women who had continued bleeding received booster doses of TXA with a significantly $(Z=2.220$, $\mathrm{p}=0.026$ ) higher dose in control group compared to TXA group, (Fig. 2).

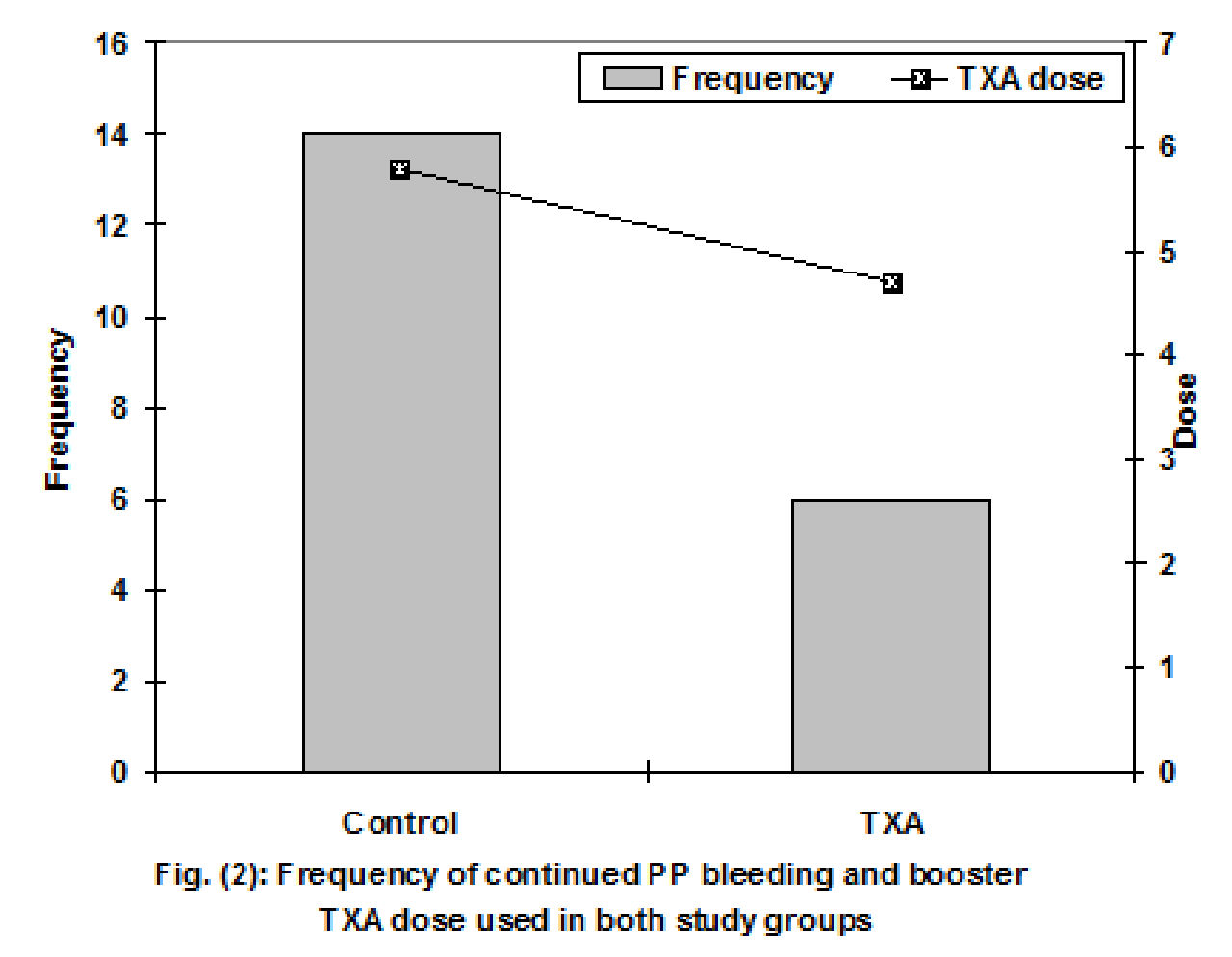

significantly ( $Z=1.204 \& 1.508$, respectively, $p>0.05$ ) different between both groups. Twelve patients developed nausea and two of them had an attack of vomiting and had responded to intravenous metochlopramide. Seventeen patients had mild shivering, but no respiratory depression was recorded in either group. There was nonsignificant difference between both studied groups as regards spinal block-related side effects. No thrombotic complications were reported in TXA group.

With regard to spinal related side effects, nausea occur in 32 patients $(18.3 \%), 17$ in control group $(53.1 \%)$ and 15 patients in tranexamic group $(46.8 \%)$ only 5 patients develop vomiting and respond to single dose of onadnosterone $(4 \mathrm{mg}) .40$ patients $(22.9 \%)$ in the study develop shivering, 22 patients $(55 \%)$ in control group and 18 patients (45\%) in tranexamic group and patients responding to 25 $\mathrm{mg}$ pethidine I.V. There is no significant difference between 2 groups in spinal related side effects.

\section{DISCUSSION}

The current study was bi-armed aiming to evaluate the impact of fluid coload on spinal block related hypotension and to determine the effect of TXA therapy according to the used protocol on intraoperative and postoperative bleeding. As regards hypotension, the commonest complication of spinal anesthesia, the ideal timing for fluid therapy to combat for occurrence and severity of hypotension is still a matter of debate; Dyer et al. ${ }^{[22]}$ compared preload and coload during spinal anesthesia for CS and found coload group significantly required a lower median dose and a lower median number of ephedrine doses for the treatment of maternal hypotension pre-delivery. On the other hand, Siddik-Sayyid et al. [23] reported non-significantly higher incidence of hypotension (68\% vs. $75 \%)$ and of severe hypotension ( $16 \%$ vs. $22 \%$ ) with coloading compared to preloading. On contrary and in support of coloading, Banerjee et al. ${ }^{[24]}$ retrieved randomized controlled trials that compared a fluid preload with coload in patients undergoing spinal anesthesia for elective CS and reported non-significantly reduced frequency of hypotension with coloading.

In support of fluid co-loading, the current study reported a frequency of hypotension of $30.7 \%$ and of sever hypotension $(<80 \mathrm{mmHg}$ ) of $22.6 \%$ of those had hypotension. Such effect of coloading was also manifested as reduction of the frequency and total dose of ephedrine used and was beneficial for maintaining good fetal perfusion which reflected as high Apgar scores detected at one and five minutes after delivery. 
The difference reported between the current study and that reported in literature could be attributed to the used dose and rate of fluid therapy. In hand with the obtained results and this assumption; Loubert [25] found crystalloid preload is not as effective as either of colloid preload; colloid coload or crystalloid coload which are equivalent for the prevention of maternal hypotension and a reduced need for vasopressors. Recently, Mercier et al. ${ }^{[26]}$ documented that crystalloid preloading is clinically ineffective and should be abandoned, while crystalloid coloading at the onset of sympathetic blockade is better, but its efficacy may depend on the volume infused and the speed of administration.

Concerning control of bleeding, the other arm of the study, patients received TXA prophylaxis and continuous therapy showed significantly lower amount of intraoperative and postoperative bleeding with lower frequency of parturient had continued PP bleeding for $>2$-hr PP and so required significantly lower booster TXA dose and consumed significantly lower dose of uterotonic drugs compared to control group. These results go in hand with that previously reported in literature concerning the prophylactic use of TXA; Gai et al. ${ }^{[27]}$ found intravenous TXA given immediately before CS statistically reduces the extent of bleeding from placental delivery to $2 \mathrm{~h}$ postpartum and its use was not associated with any side effects or complications. Sekhavat et al. ${ }^{[28]}$ also, found TXA statistically reduced blood loss from end to $2 \mathrm{~h}$ after $\mathrm{CS}$ and its use was not associated with any side effects or complications.

Movafegh et al. ${ }^{[29]}$ tried intravenous injection of 10 $\mathrm{mg} / \mathrm{kg}$ of TXA before incision and found mean blood loss was significantly less in TXA group compared with the control group for both intraoperative and postoperative bleeding and the total dose of oxytocin used was significantly lower with TXA. Gungorduk et $a l .{ }^{[30]}$ found TXA significantly reduced bleeding during $\mathrm{CS}$, the percentage of patients with blood loss $>1000$ $\mathrm{ml}$, and the need for additional uterotonic agents.

Abdel-Aleem et al. ${ }^{[31]}$ used one gm tranexamic acid slowly intravenously over $10 \mathrm{~min}$ before elective CS and found the mean drop in hematocrit and hemoglobin levels were significantly lower in TXA group than in control group and concluded that pre-operative TXA is associated with reduced blood loss during and after elective CS. Sentürk et al. ${ }^{\left[{ }^{32]}\right.}$ used TXA $20 \mathrm{cc}$ injected $10 \mathrm{~min}$ before the start of CS and found TXA reduced intraoperative and postoperative blood loss without concomitant complications caused by TA such as venous thromboembolism, gastrointestinal problems
$\mathrm{Xu}$ et al. ${ }^{[33]}$ tried intravenous injection of $10 \mathrm{mg} / \mathrm{kg}$ TXA immediately before CS and found blood loss in the period between the end of CS and $2 \mathrm{~h}$ postpartum was significantly lower in the TXA group than in the control group and PP hemorrhage stopped in $75.6 \%$ of controls and in $92 \%$ in TXA group with significant difference in favor of TXA.

In contrary to the obtained results and these reported in literature; Shahid \& Khan ${ }^{[34]}$ found TXA injection significantly reduced the amount of blood loss during SC, but it did not significantly reduce blood loss after $\mathrm{CS}$ and its use was not associated with any side effects or complication like thrombosis. This difference could be attributed to the dose used or the timing of injection and may indicate the need and manifest the advantage of the continued use of TXA infusion similar to the applied current study protocol.

\section{CONCLUSION}

It could be concluded that preoperative administration of TXA as a loading dose and continuous infusion significantly reduced perioperative blood loss with significant reduction of consumption of TXA booster doses and utrotonics without affecting safety. Fluid coloading allowed reduction of the frequency and extent of hypotension concomitant with spinal anesthesia. Wherever possible, estimation of amount of blood loss depending on the change of hematocrite value is preferable for determination of amount of blood loss and the requirement for replacement therapy.

\section{ACKNOWLEDGMENT}

The authors would like to thank residents and nurses of the Departments of Obstetrics \& Gynecology and Anesthesia, Benha Faculty of Medicine for their role in patients follow up and collection of postoperative data. The authors also thank professor Abd Elmoniem Abd El Fatah Younis (Community and Public-Health Department, Benha Faculty of Medicine) for performing the statistics of this study.

\section{CONFLICT OF INTEREST}

There is no conflict of interest.

\section{REFERENCES}

1. Mercier FJ, Bonnet MP, De la Dorie A, Moufouki M, Banu F, Hanaf A, Edouard D, Roger-Christoph S: Spinal anaesthesia for caesarean section: fluid loading, vasopressors and hypotension. Ann Fr Anesth Reanim. 2007;26(7-8):688-93. 
2. Yokoyama K, Suzuki M, Shimada Y, Matsushima T, Bito H, Sakamoto A: Effect of administration of pre-warmed intravenous fluids on the frequency of hypothermia following spinal anesthesia for Cesarean delivery. J Clin Anesth. 2009; 21(4):242 -8.

3. Jo YY, Kim JY, Kim JS, Kwon Y, Shin CS: The effect of ephedrine on intraoperative hypothermia. Korean J Anesthesiol. 2011; 60(4):250- 4.

4. Gulhas N, Tekdemir D, Durmus M, Yucel A, Erdil FA, Yologlu S, Ersoy MO: The effects of ephedrine on maternal hypothermia in caesarean sections: a double blind randomized clinical trial. Eur Rev Med Pharmacol Sci. 2013;17(15):2051-8.

5. Ramadani H: Cesarean section intraoperative blood loss and mode of placental separation. Int J Gynaecol Obstet. 2004;87(2):114-8.

6. Caughey AB, Sundaram V, Kaimal AJ, Cheng YW, Gienger A, Little SE, Lee JF, Wong L, Shaffer BL, Tran SH, Padula A, McDonald KM, Long EF, Owens DK, Bravata DM: Maternal and neonatal outcomes of elective induction of labor. Evid Rep Technol Assess (Full Rep). 2009;(176):1-257.

7. Bodner K, Wierrani F, Grünberger W, Bodner-Adler $\mathrm{B}$ : Influence of the mode of delivery on maternal and neonatal outcomes: a comparison between elective cesarean section and planned vaginal delivery in a low-risk obstetric population. Arch Gynecol Obstet. 2011; 283(6):1193-8.

8. Sankar D, Krishnan R, Veerabahu M, Vikraman B: Evaluation of the efficacy of tranexamic acid on blood loss in orthognathic surgery. A prospective, randomized clinical study. Int J Oral Maxillofac Surg. 2012; 41(6):713-7.

9. Wind TC, Barfield WR, Moskal JT: The Effect of Tranexamic Acid on Blood Loss and Transfusion Rate in Primary Total Knee Arthroplasty. J Arthroplasty. 2013: S0883-5403(13)00103-4.

10. Roberts I, Shakur H, Coats T, Hunt B, Balogun E, Barnetson L, Cook L, Kawahara T, Perel P, PrietoMerino D, Ramos M, Cairns J, Guerriero C: The CRASH-2 trial: a randomised controlled trial and economic evaluation of the effects of tranexamic acid on death, vascular occlusive events and transfusion requirement in bleeding trauma patients. Health Technol Assess. 2013;17(10):1-79.

11. Vijay BS, Bedi V, Mitra S, Das B: Role of tranexamic acid in reducing postoperative blood loss and transfusion requirement in patients undergoing hip and femoral surgeries. Saudi J Anaesth. 2013; 7(1):29-32.
12. Tamilselvan P, Fernando R, Bray J, Sodhi M, Columb M: The effects of crystalloid and colloid preload on cardiac output in the parturient undergoing planned cesarean delivery under spinal anesthesia: a randomized trial. Anesth Analg. 2009; 109(6):1916-21.

13. Tyagi A, Kumar A, Girotra G, Sethi AK: Combined spinal epidural and epidural volume extension: Interaction of patient position and hyperbaric bupivacaine. J Anaesthesiol Clin Pharmacol. 2011;27(4):459-64.

14. Bromage PR, Burfoot MF, Crowell DE, Pettigrew RT: Quality of epidural blockade: I Influence of physical factors. Br. J. Anaeth., 1964; 36: 342-52.

15. Buchman MI: Blood loss during gynecological operations. Am J Obstet. Gynecol., 1953; 65: 53-64

16. Gross JB: Estimating Allowable Blood Loss: Corrected for Dilution. Anesthesiology 1983; 58(3): 277-80.

17. Mc Swiney MM, O'Farrell D, Joshi GP, Mc Carroll SM: Blood Transfusion in Total Hip Athroplasty: Guidelines to eliminate overtransfusion. Can J Anaesth 1993; 40(3): 222-26.

18. Eipe N, Ponniah M: Perioperative blood loss assessment- How accurate? Indian J Anaesth., 2006; 50(1): $35-8$.

19. Kraemer HC, Theimann S: How many subjects? Statistical power analysis in research; 1987, Newbury Park, CA: Sage.

20. Murphy KR, Myors B: Statistical Power Analysis: A Simple and General Model for Traditional and Modern Hypothesis Tests; 2003, 2nd Ed., Lawrence Erlbaum Associates, Inc.

21. Day SJ, Graham DF: Sample size and power for comparing two or more treatment groups in clinical trials. BMJ, 1989; 299: 663-5.

22. Dyer RA, Farina Z, Joubert IA, Du Toit P, Meyer M, Torr G, Wells K, James MF: Crystalloid preload versus rapid crystalloid administration after induction of spinal anesthesia (co-load) for elective caesarean section. Anaesth Intensive Care. 2004;32(3):351-7.

23. Siddik-Sayyid SM, Nasr VG, Taha SK, Zbeide RA, Shehade JM, Al Alami AA, Mokadem FH, Abdallah FW, Baraka AS, Aouad MT: A randomized trial comparing colloid preload to coload during spinal anesthesia for elective cesarean delivery. Anesth Analg. 2009;109(4):1219-24. 
24. Banerjee A, Stocche RM, Angle P, Halpern SH: Preload or co-load for spinal anesthesia for elective Cesarean delivery: a meta-analysis. Can J Anaesth. 2010; 57(1):24-31.

25. Loubert C: Fluid and vasopressor management for Cesarean delivery under spinal anesthesia: continuing professional development. Can J Anaesth. 2012;59(6):604-19.

26. Mercier FJ, Augè M, Hoffmann C, Fischer C, Le Gouez A: Maternal hypotension during spinal anesthesia for caesarean delivery. Minerva Anestesiol. 2013;79(1):62-73

27. Gai MY, Wu LF, Su QF, Tatsumoto K: Clinical observation of blood loss reduced by tranexamic acid during and after caesarian section: a multi-center, randomized trial. Eur J Obstet Gynecol Reprod Biol. 2004; 112(2):154-7.

28. Sekhavat L, Tabatabaii A, Dalili M, Farajkhoda T, Tafti AD: Efficacy of tranexamic acid in reducing blood loss after cesarean section. J Matern Fetal Neonatal Med. 2009; 22(1):72-5.

29. Movafegh A, Eslamian L, Dorabadi A: Effect of intravenous tranexamic acid administration on blood loss during and after cesarean delivery. Int J Gynaecol
Obstet. 2011; 115(3):224-6.

30. Gungorduk K, Yıldırım G, Asıcığglu O, Gungorduk OC, Sudolmus S, Ark C: Efficacy of intravenous tranexamic acid in reducing blood loss after elective cesarean section: a prospective, randomized, doubleblind, placebo-controlled study. Am J Perinatol. 2011;28(3):233-40.

31. Abdel-Aleem H, Alhusaini TK, Abdel-Aleem MA, Menoufy M, Gülmezoglu AM: Effectiveness of tranexamic acid on blood loss in patients undergoing elective cesarean section: randomized clinical trial. J Matern Fetal Neonatal Med. 2013; Epub ahead of print.

32. Sentürk MB, Cakmak Y, Yildiz G, Yildiz P: Tranexamic acid for cesarean section: a double-blind, placebo-controlled, randomized clinical trial. Arch Gynecol Obstet. 2013;287(4):641-5.

33. $\mathrm{Xu} \mathrm{J}, \mathrm{Gao} \mathrm{W}, \mathrm{Ju} \mathrm{Y}$ : Tranexamic acid for the prevention of postpartum hemorrhage after cesarean section: a double-blind randomization trial. Arch Gynecol Obstet. 2013; 287(3):463-8.

34. Shahid A, Khan A: Tranexamic acid in decreasing blood loss during and after caesarean section. J Coll Physicians Surg Pak. 2013; 23(7):459-62. 